\title{
An Approach to Inundation Simulation in Large River Basins Using the Triangle Finite Difference Method
}

\author{
X. H. Zhang ${ }^{1 *}$, H. Ishidaira ${ }^{1}$, K. Takeuchi ${ }^{1}$, Z. X. Xu ${ }^{1}$ and X. W. Zhang ${ }^{2}$ \\ ${ }^{1}$ Department of Civil and Environmental Engineering, University of Yamanashi, Kofu 400-8511, Japan \\ ${ }^{2}$ Department of Planning, Ministry of Water Resources, Beijing 100053, China
}

\begin{abstract}
Disastrous flooding and inundation events have drawn much attention in regional sustainable development due to extensive life loss and property damage they have caused. To support river basin management practice, it is necessary to simulate flood inundation such that associated risks can be integrated into decision-making processes. Flood inundation is generally simulated by the 2-dimensional Saint Venant equations (2D SVEs), in which inertia terms are neglected in most cases, particularly in large floodplains. In this study, a diffusive wave model discretized by an implicit numerical scheme of the irregular triangle finite difference method (TFDM) was developed to examine the effect of arbitrary inner and outer boundaries within a floodplain. A case study has been conducted in the Fuefuki River basin in central Japan. Comparisons with simulation using complete 2D SVEs are made in this study. Results indicate that both the diffusive wave model and its TFDM numerical scheme are effective and accurate in the simulation of flood inundation.
\end{abstract}

Keywords: Inundated area, inundated water depth, inundation simulation, irregular triangle mesh, St. Venant equation

\section{Introduction}

Disastrous flooding events have increased greatly due to aggressive consumption of natural resources and global warming effects. The number of large flood disasters in the nine years from 1990 to 1998 was higher than those in the three-and-half decades between 1950 and 1985. The global loss from floods has grown to billions of dollars annually. The flood inundation due to dike-breaks or overtopping is mostly attributable to the damages as many metropolitans are protected by these dikes. Flood inundation in the context of this study is defined as the transportation of 2D flooding flows due to dike-break or overtopping. It is demanded that flood risk assessment must be integrated into decision analyses for environmental management and sustainable development of watersheds. Flood-inundation simulation is believed to be a necessary part of contemporary management practices.

Flood inundation flows are commonly expressed by $2 \mathrm{D}$ SVEs. These equations describe flows in vertically wellmixed water bodies where horizontal length scales are much greater than water depths, which belong to long wavelength phenomena. They were obtained by assuming a hydrostatic pressure distribution and a uniform velocity profile in vertical direction. By integrating the Navier-Stokes equations along water depths, a three-dimensional free boundary problem is reduced to a $2 \mathrm{D}$ fixed boundary problem with primary variables including water depths and velocities averaged vertically. The 2D SVEs can be used to study many physical phenomena

\footnotetext{
* Corresponding author: xinhuazhi@ccn.yamanashi.ac.jp
}

of interest, such as storm surges, tidal fluctuations, tsunami waves, and can also be coupled with transport equations to simulate transports of sediments or other species because of its easiness in the coupling (Aizinger \& Dawson, 2002). However, some large river basins such as the South Florida basin in the US and the lower Yellow River basin in China are characterized by large areal extents, low slopes, widespread ponds, tributaries, and slow regional flow dynamics. These properties allow an approximation of 2D SVEs based on the theoretical conditions proposed by Ponce et al. (1978). Among available approximation models, kinematic wave models are inadequate for these cases because they neglect backwater effects completely. Diffusion wave models have been found to be capable of simulating varieties of natural flow conditions successfully. For example, hydrologic conditions in Everglades, USA have been successfully simulated with both NSM (Natural System Model) and SFWMM (South Florida Water Management Model) in which the 2D diffusion wave models are utilized (Fennema et al., 1994). Akan \& Yen (1981) applied diffusion wave flood routing in channel networks to deal with the mutual backwater effects existing among the channel branches joining at a junction. Xanthopoulos and Koutitas (1976) have applied such a model to simulate 2D flood wave propagation due to dam failure. Hromadka \& Lai (1985) also used a 2D diffusive wave model discretized by a finite difference method with uniform rectangular meshes to estimate the effects of the diverging flood flow due to dam-break. The results of their study showed that the 2D diffusion dam-break model could provide fast, economical, and practical estimates of flood depths, areas and time of propagation. The diffusive wave 
model can be a good approximation of the dynamic wave model because of its ability to accommodate both backwater effects and unsteady flow. However, the both of them are neglected in the HEC-2 floodplain analysis (Hromadka II et al., 1989).

Dike break and dam failure have similar mechanisms of breaking, but dynamic property of flooding flow due to dike-break is not significant compared with dam-break flows. The dam-break flows are usually confined within a narrow valley with the characteristics of shock properties existing at the waterfront and the dynamic component is significantly large. The flooding flows due to dike-break, on the other hand, propagate freely in a large floodplain which make the flows to be diffusive, and are dominated by forces of gravity, pressure, and friction. Because good results have been obtained from 1D diffusive flow model for the problems of dam failure, such a model should be more reasonable to be applied to the $2 \mathrm{D}$ inundation simulation due to dike break. The main concerns are the inundated areas and water depths, especially for the purposes of flood risk assessment and management within a river basin.

Complexities of floodplains and arbitrarily geometrical structures within floodplains, including their boundaries both inside and outside of study regions, for instance, roads, drainage networks, farmlands, forests, and buildings, may also significantly affect accuracies of simulation results. An ideal model for the simulation of $2 \mathrm{D}$ overland flow is expected to be capable of handling water bodies of arbitrary shapes and using flexible ranges in temporal and spatial scales to meet accuracy requirements at different locations and times. The uniformly structured mesh system in the Cartesian coordinate can easily generate computational meshes. The flooding conditions in both inundated areas and water depths can be simulated efficiently with digital elevation models (DEM) of different resolutions. But the effects of buildings and other artificial structures on the accuracy of the simulation cannot be considered accordingly. Much effort has been made to improve this type of methods. For instance, Hromadka II et al (1989) introduced both flow-path reduction factor and effective grid area to consider influences of buildings within floodplain; Suetsugi and Kuriki (1998) integrated this effect in the manning coefficient through a relationship among ratios of buildings, water-depth, and roughness coefficient. Their methods can improve the accuracy undoubtedly, but the geometrical influence of arbitrary structures cannot be integrated into the simulation. A possible approach to overcoming this difficulty is the appli- cation of unstructured grid systems with the calculation elements such as irregular triangles and quadrilaterals.

The merits of unstructured grids are that the lack of grid structure makes it possible to mesh arbitrary geometries with relative easiness, and offers capability to locally adapt the grid by simply adding extra grid points where they are needed, to improve the accuracy of the computation without incurring the penalties associated with global refinement (Anderson \& Bonhaus, 1994; Holmes \& Connell, 1989). The control volumes are formed by dividing the study region into a finite number of irregular elements such as triangles or quadrilaterals. Then, solutions are obtained based on these control volumes.

As above noted, most numerical algorithms in flood inundation models are based on the uniform rectangular mesh system, and they can hardly integrate the influence of geometrical and topographical complexities of floodplains into the simulation. For the purpose of surmounting the limitation, a simple and completely implicit scheme of TFDM based on irregular triangle meshes is developed in this work. The first objective of this study is to investigate the possibility of applying diffusive wave model to the simulation of overland inundation flow due to dike-break or overtopping in large river basins. Secondly it is to testify the effectiveness of the implicit TFDM scheme for the diffusive wave model. Also if the above two objectives prove to be feasible, the model and its numerical scheme would be applied to the simulation of flood inundation on complex land-covers of floodplains in large river basins.

\section{Governing Equations of Diffusive wave Model}

The governing equation of 2D SVEs can be written in the form of fluxes as follows:

$$
\begin{aligned}
& \frac{\partial h}{\partial t}+\frac{\partial M}{\partial x}+\frac{\partial N}{\partial y}-\underbrace{R F+I N+E T}_{\text {sources }}=0 \\
& \frac{\partial M}{\partial t}+\frac{\partial(u M)}{\partial x}+\frac{\partial(v M)}{\partial y}=-g h \frac{\partial H}{\partial x}-\frac{\tau_{b x}}{\rho} \\
& \frac{\partial N}{\partial t}+\frac{\partial(u N)}{\partial x}+\frac{\partial(v N)}{\partial y}=-g h \frac{\partial H}{\partial y}-\frac{\tau_{b y}}{\rho}
\end{aligned}
$$

where $h$ is the inundated water depth; $M(=u h)$ and $N(=v h)$ are discharge flux per unit width in $x$ - and $y$-orientation, respectively; $R F=$ rainfall; $I N=$ infiltration; and $E T=$ evapotranspiration. Here, $R F, I N$ and $E T$ are regarded as source terms which are expressed in unit of length/time; $u$ and $v$ are flow velocity in $x$ and $y$ directions; $g$ is gravitational acceleration; $H$ is surface water level which equals to $Z_{\mathrm{b}}+h$, here, $Z_{\mathrm{b}}$ is the ground elevation. $\rho$ is the mass density of water flow; $\tau_{b x}, \tau_{b y}$ are ground shear stresses in $x$ and $y$ directions, respectively. They can be estimated by the equations as follows:

$$
\begin{aligned}
& \tau_{b x} / \rho=g n^{2} u \sqrt{u^{2}+v^{2}} / h^{1 / 3} \\
& \tau_{b y} / \rho=g n^{2} v \sqrt{u^{2}+v^{2}} / h^{1 / 3}
\end{aligned}
$$

where $n$ is the Manning's roughness coefficient.

Based on the results obtained by Xanthopoulos and 
Koutitas (1976), Hromadka (1989, 1986), Lal (1998, 2000), and Ponce (1978), it is reasonable to ignore the inertia terms in the complete 2D SVEs for the simulation of overland flows. Equations (2) and (3) can be written as:

$$
\begin{aligned}
& -g h \frac{\partial H}{\partial x}=\frac{\tau_{b x}}{\rho}=\frac{g n^{2} u|\vec{V}|}{h^{1 / 3}} \\
& -g h \frac{\partial H}{\partial y}=\frac{\tau_{b y}}{\rho}=\frac{g n^{2} v|\vec{V}|}{h^{1 / 3}}
\end{aligned}
$$

Considering Manning's Equation for the magnitude of velocity vectors and assuming that the friction slope $S_{f}$ is equal to the slope of water surface $S_{s}$, i.e.

$$
\begin{aligned}
& |\vec{V}|=\sqrt{u^{2}+v^{2}}=\frac{1}{n} h^{2 / 3} S_{f}^{1 / 2} \\
& S_{f}=S_{s}=\sqrt{(\partial H / \partial x)^{2}+(\partial H / \partial y)^{2}}
\end{aligned}
$$

The velocity components $u$ and $v$ can be rearranged as the following form on the basis of Manning's Equation:

$$
\left.\begin{array}{c}
u=-\frac{K}{h} \frac{\partial H}{\partial x}, \quad v=-\frac{K}{h} \frac{\partial H}{\partial y} \\
K=\frac{h^{5 / 3}}{n \sqrt{S_{s}}}
\end{array}\right\}
$$

The fluxes per unit width in $\mathrm{x}$ - and $\mathrm{y}$-directions will be simplified as:

$$
M=-K \frac{\partial H}{\partial x}, \quad N=-K \frac{\partial H}{\partial y}
$$

By instituting the fluxes of Equation (1) with Equation (11), the diffusive wave model can be rewritten as:

$$
\left.\begin{array}{l}
\frac{\partial H}{\partial t}=\frac{\partial}{\partial x}\left(K \frac{\partial H}{\partial X}\right)+\frac{\partial}{\partial y}\left(K \frac{\partial H}{\partial y}\right)+\underbrace{R F-I N-E T}_{\text {Sources }} \\
\text { where, } \quad K=\frac{h^{5 / 3}}{n \sqrt{S_{s}}}
\end{array}\right\}
$$

The coefficient $K$ is the constant of hydraulic conductivity in ground water equations. In the diffusive overland flow equations, $K$ is an important parameter to be able to linearize and simplify the equations. As expressed in the equations, $K$ must be kept in a reasonable limit for the wetting or drying situations. In this paper, $K$ is treated in the same way as that of Lal (1998):
$\begin{cases}K=\frac{h^{5 / 3}}{n \sqrt{S_{S}}} & \text { for }\left|S_{S}\right| \geq \delta \quad \text { and } \quad h \geq h_{\text {min }} \\ K=0 & \text { otherwise }\end{cases}$

In this study, threshold of water depth $h_{\min }=0.001 \mathrm{~m}$ is used to determine frontier of flooding flows. In other words, the drying and wetting conditions can be judged based on the value. A very small $\delta=1.0 \times 10^{-7}$ is also used to control $K$ within a finite reasonable range when the slope of the water surface is an infinitesimal value.

\section{Implicit TFDM Numerical Scheme of Diffusive Wave Model}

Generally, the finite difference method (FDM) with regular mesh has the advantages of theoretical clearness, easiness in mesh generation, and simplicity in computer programming. However, it will confront a complex situation if irregular boundaries exist in a study region. In order to overcome this problem, the irregular triangle finite difference method (TFDM) is proposed in this study. The generation of triangle meshes has to be done as follows: 1) the physical domain has to be completely covered by the meshes; 2) there must be no free space left between the meshes, and the meshes should not be overlapped; 3) any inner angle should not be greater than $90^{\circ}$.

Based on these basic requirements for mesh generation, the overland flooding flow can be simulated through the irregular triangle meshes as displayed in Figure 1.

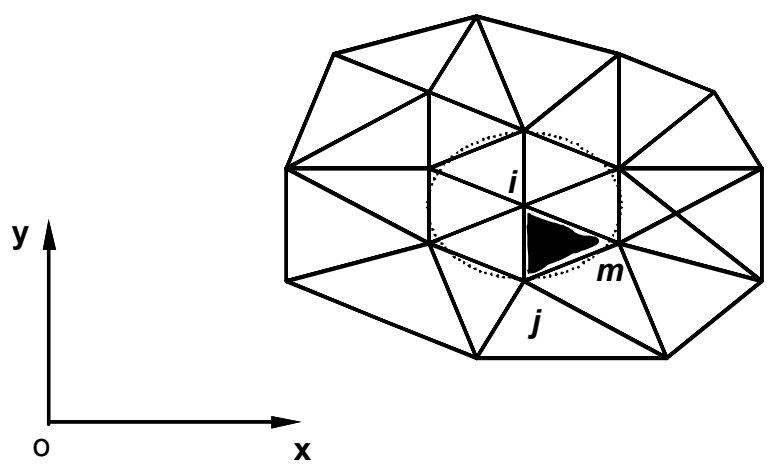

Figure 1. Subdivisions of the study area into irregular triangle meshes.

Because the governing equation of diffusive wave model is almost the same as that of the basic governing differential equations of groundwater movements, Darcy's Law can also be applied to the discretization process of the governing equation (12). The discharge flux has a direct relationship with the hydraulic gradients based on Darcy's Law. Therefore, this relationship can be used as a guideline to design the control volume. For this purpose, the control volumes designed in this 
paper have three prominent kinds of characteristics over others: 1) cell-vertex scheme with boun- dary sides of the control volume perpendicular to the sides/ edges of meshes surrounding a given node; 2) the direction of hydraulic gradients in the calculation parallels with the unit outward normal vectors at the boundary sides of control volumes, so the tedious projection calculation of fluxes into the direction of normal vectors can be avoided; and 3) a completely implicit scheme making it suitable for enlarging time-step in simulations for longer time span.

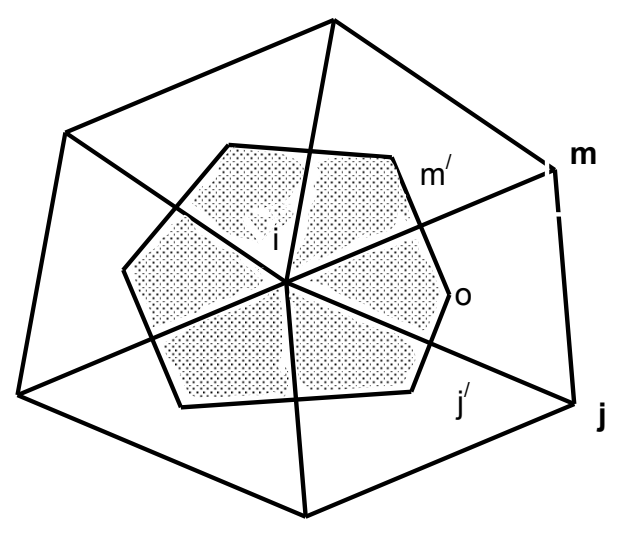

Figure 2. Effective control volume contributed to the water level change at node $i$ shaded by dots.

The control volume at each vertex is designed by drawing a perpendicular line through the edge-mid-point of all cells sharing a given node. An exemplary control volume is displayed in Figure 2. By integrating Equation (12) over a control volume $\Omega$ and using the Gauss theorem, the basic equation of the TFDM can be expressed as:

$\iint_{\Omega} \frac{\partial H}{\partial t} d \omega=\int_{\Gamma_{\Omega}} \mathrm{F}(H) \cdot \mathrm{n} d L+\iint_{\Omega} S d \omega$

in which $n=$ a unit outward vector normal to the boundary $\Gamma_{\Omega}$; $d \omega$ and $d L$ are the differential area of the control volume and the differential length of the boundary sides of the control volume, respectively. The integrand $F(H) \bullet n$ is the normal flux vector which can be obtained by Darcy's Law as mentioned before due to the properties of the control volume in this study. By discretizing Equation (14), the basic numerical equation of the TFDM is

$A_{i} \frac{\partial H_{i}}{\partial t}=\sum_{s=1}^{m} q_{i}^{s} L_{i}^{s}+A_{i} S_{i}$

where $A_{i}=$ area of control volume $\Omega$ at node $i ; m=$ number of boundary sides of control volume; $q_{i}^{s}=$ discharge flux into control volume through boundary side $s ; L_{i}^{s}=$ length of boundary side $s ; S_{i}=$ sources flowing into or out of control volume. Physical meanings of Equation (15) can be explained as balance of water quantity within the control volume. The first term at the right side of the equation represents the total quantity of the lateral water inflow. The second term on right side of the equation represents supply quantity in vertical direction of the control volume due to recharging from rainfall and drainages from infiltration or pumping. The only term on left side of the equation (15) means total quantity variation within the control volume. To make the equation easier to understand, Equation (15) can be rewritten as:

$\Delta Q_{i, \text { storage }}=\Delta Q_{i, \text { lateral }}+\Delta Q_{i, \text { vertical }}$

In order to get the complete implicit TFDM numerical schemes, three terms in Equation (16) will be discussed in further details in the following sections.

\subsection{Estimation of Lateral Quantity Flowing into Control Volume $\Delta \mathbf{Q}_{i, \text { Lateral }}$}

In this TFDM scheme, the most important and difficult task is the estimation of lateral quantity flowing into the control volume. For the convenience of explanations, triangle $\Delta i j m$, as shown in Figure 3, is assumed as triangle $\beta$ and all vertices of the triangles are numbered in a counter-clock wise order.

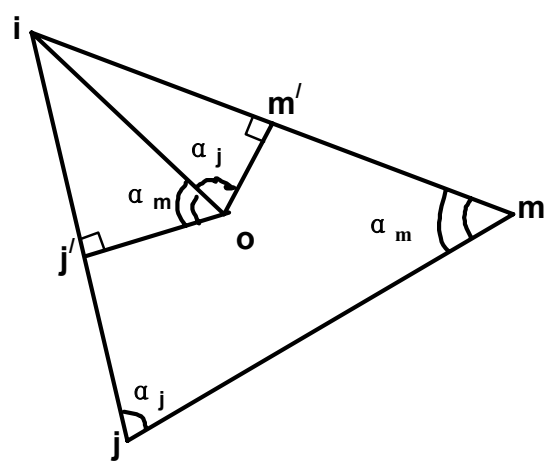

Figure 3. Contributions of triangle $\Delta_{\mathrm{ijm}}$ to the total effective control volume of node $i$.

$\Delta Q_{i, \text { lateral }}^{(\beta)}=K_{i j} \frac{H_{j}-H_{i}}{i j} o j^{\prime}+K_{i m} \frac{H_{m}-H_{i}}{i m} o m^{\prime}$

From the geometrical relationship as indicated in Figure 3 , the function can be explained as: $\cot \alpha_{\mathrm{m}}=2 o j^{\prime} / i j$ and $\cot \alpha_{\mathrm{j}}=2 o m^{\prime} / \mathrm{im}$ because both $o j^{\prime}$ and $o m^{\prime}$ are perpendicular 
lines drawn from middle of lines $i j$ and $i m$, respectively. Thus Equation (17) can be rearranged as follows:

$$
\Delta Q_{i, \text { lateral }}^{(\beta)}=\frac{1}{2} K_{i j}\left(H_{j}-H_{i}\right) \cot \alpha_{m}+\frac{1}{2} K_{i m}\left(H_{m}-H_{i}\right) \cot \alpha_{j}
$$

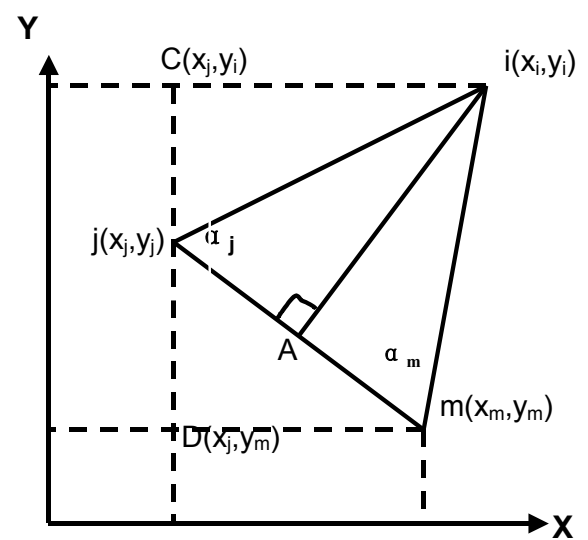

Figure 4. Inner angles and coordinates of triangular element $\beta$.

From Figure 4, values of triangular function in Equation (18) can be given as:

$\cot \alpha_{j}=\frac{j A}{i A}=\frac{j A \cdot j m}{i A \cdot j m}=\frac{j A \cdot j m}{2 A^{\beta}}$

where $j A$ and $j m$ are the lengths of lines, while $A^{\beta}$ is the area of triangle $\beta$. If $j A$ can be expressed by sides of triangle $\beta$, the triangular functions can be simplified as a function of coordinates of the vertices. In Figure 4, we can get a relationship as:

$j A \cdot j m=\frac{i j^{2}-i m^{2}+j m^{2}}{2}$

Equation (19) thus can be expressed by the known sides of triangle $\beta$, i.e.

$\cot \alpha_{j}=\frac{i j^{2}-i m^{2}+j m^{2}}{4 A^{\beta}}$

Considering the coordinates of each vertex of triangle $\beta$, the function can be further represented as:

$\cot \alpha_{j}=\frac{1}{2 A^{\beta}}\left[\left(x_{i}-x_{j}\right)\left(x_{m}-x_{j}\right)+\left(y_{i}-y_{j}\right)\left(y_{m}-y_{j}\right)\right]$

For the same reason, $\cot \alpha_{m}=\frac{1}{2 A^{\beta}}\left[\left(x_{j}-x_{m}\right)\left(x_{i}-x_{m}\right)+\left(y_{j}-y_{m}\right)\left(y_{i}-y_{m}\right)\right]$

Supposing that

$\left\{\begin{array}{l}b_{i}=y_{j}-y_{m} \\ b_{j}=y_{m}-y_{i} \\ b_{m}=y_{i}-y_{j}\end{array} \quad\left\{\begin{array}{l}c_{i}=x_{m}-x_{j} \\ c_{j}=x_{i}-x_{m} \\ c_{m}=x_{j}-x_{i}\end{array}\right.\right.$

are used to represent relationships among coordinates of the vertices, and Equations (22) and (23) can be rewritten as follows:

$\left\{\begin{array}{l}\cot _{j}=-\frac{1}{2 A^{\beta}}\left(b_{i} b_{m}+c_{i} c_{m}\right) \\ \cot _{m}=-\frac{1}{2 A^{\beta}}\left(b_{i} b_{j}+c_{i} c_{j}\right)\end{array}\right.$

Substituting Equation (25) into Equation (18), lateral discharge flux flowing into the control volume by triangle $\beta$ can be obtained as:

$$
\begin{aligned}
\Delta Q_{i, \text { lateral }}^{\beta}= & -\frac{1}{4 \mathrm{~A}^{\beta}}\left[K_{i j}\left(H_{j}-H_{i}\right)\left(b_{i} b_{j}+c_{i} c_{j}\right)\right. \\
& \left.+K_{i m}\left(H_{m}-H_{i}\right)\left(b_{i} b_{m}+c_{i} c_{m}\right)\right]
\end{aligned}
$$

Because the total number of unstructured triangular elements surrounding node $i$ is $m$, lateral quantity flowing into the control volume can be finally obtained by the following equation (27).

$$
\begin{aligned}
\Delta Q_{i, \text { lateral }}= & \sum_{\beta=1}^{m} \Delta Q_{i, \text { lateral }}^{\beta} \\
= & -\sum_{\beta=1}^{m} \frac{1}{4 \mathrm{~A}^{\beta}}\left[K_{i j}\left(H_{j}-H_{i}\right)\left(b_{i} b_{j}+c_{i} c_{j}\right)\right. \\
& \left.+K_{i m}\left(H_{m}-H_{i}\right)\left(b_{i} b_{m}+c_{i} c_{m}\right)\right]
\end{aligned}
$$

\subsection{Addition of Vertical Quantity to Control Volume $\Delta \mathbf{Q}_{i, \text { Vertical }}$}

The vertical quantity added to the control volume refers to the rainfall supplies, pump drainage, and other. If the density of these kinds of supplies or drainages is $W_{i}$ and effective area of the control volume around node $i$ is $A_{i}$, then vertical quantity added to the control volume can be described as:

$$
\Delta Q_{i, \text { vertical }}=A_{i} W_{i}
$$


So far, the effective area $A_{i}$ of the control volume at node $i$ has yet to be given. For this purpose, Figure 3 can be used to correlate again. Because the effective area of triangle $\beta$ to the whole control volume is made up by the quadrilateral element $i j^{\prime} \mathrm{om}^{\prime}$ which consists of $\Delta i j^{\prime} O$ and $\Delta i o m^{\prime}$, the effective area in this triangular element can be easily achieved. For example, the area of $\Delta i j^{\prime} O$ can be written as:

$$
\left\{\begin{array}{l}
\because i j^{2}=\left(x_{i}-x_{j}\right)^{2}+\left(y_{i}-y_{j}\right)^{2}=b_{m}^{2}+c_{m}^{2} \\
\therefore A_{i}^{\Delta i j^{\prime} o}=\frac{1}{4} \frac{i j}{2} o j^{\prime}=\frac{i j^{2}}{8} \cot \alpha_{m} \\
\quad=-\frac{1}{16 A_{i}{ }^{\beta}}\left(b_{m}^{2}+c_{m}^{2}\right)\left(b_{i} b_{j}+c_{i} c_{j}\right)
\end{array}\right.
$$

For the same reason, the area of $\Delta i \mathrm{im}^{\prime}$ can be represented by Equation (30)

$$
\begin{aligned}
A_{i}^{\Delta i o m^{\prime}} & =\frac{1}{4} \frac{i m}{2} o m^{\prime}=\frac{i m^{2}}{8} \cot \alpha_{j} \\
& =-\frac{1}{16 A_{i}^{\beta}}\left(b_{j}^{2}+c_{j}^{2}\right)\left(b_{i} b_{m}+c_{i} c_{m}\right)
\end{aligned}
$$

The effective area of unstructured triangular element $\beta$, which is the quadrilateral element $i j^{\prime}{ }^{\prime}{ }^{\prime}$, will be the sum of these two triangles, namely:

$$
\begin{aligned}
A_{i}^{\beta}= & -\frac{1}{16 A^{\beta}}\left[\left(b_{m}^{2}+c_{m}^{2}\right)\left(b_{i} b_{j}+c_{i} c_{j}\right)\right. \\
& \left.+\left(b_{j}^{2}+c_{j}^{2}\right)\left(b_{i} b_{m}+c_{i} c_{m}\right)\right]
\end{aligned}
$$

As described before, the total number of unstructured triangular elements is $m$, the total effective area of the control volume at node $i$ can be summarized as:

$$
\begin{aligned}
A_{i}= & \sum_{\beta=1}^{m} A_{i}^{\beta} \\
= & -\sum_{\beta=1}^{m} \frac{1}{16 A^{\beta}}\left[\left(b_{m}^{2}+c_{m}^{2}\right)\left(b_{i} b_{j}+c_{i} c_{j}\right)\right. \\
& \left.+\left(b_{j}^{2}+c_{j}^{2}\right)\left(b_{i} b_{m}+c_{i} c_{m}\right)\right]
\end{aligned}
$$

Substituting Equation (32) to Equation (28), the vertical quantity added to the control volume can be given by:

$$
\begin{aligned}
\Delta Q_{i, \text { vertical }}= & A_{i} W_{i} \\
= & -W_{i} \sum_{\beta=1}^{m} \frac{1}{16 A^{\beta}}\left[\left(b_{m}^{2}+c_{m}^{2}\right)\left(b_{i} b_{j}+c_{i} c_{j}\right)\right. \\
& \left.+\left(b_{j}^{2}+c_{j}^{2}\right)\left(b_{i} b_{m}+c_{i} c_{m}\right)\right]
\end{aligned}
$$

\subsection{Storage Variation in Control Volume $\Delta Q_{i, \text { storage }}$}

Due to inflows added from both lateral and vertical directions of the control volume $\Omega_{i}$, it is supposed that the water level will be changed from $H_{i, k}$ to $H_{i, k+1}$ within a time-step $\Delta t$. This variation of storage within this control volume can be written as:

$$
\Delta Q_{i, \text { storage }}=A_{i} \frac{H_{i, k+1}-H_{i, k}}{\Delta t}
$$

\subsection{Implicit Numerical Scheme of the TFDM}

Through the above discussion, the components of water added to the control volume by different ways have been made clear. For the conciseness and adaptabilities of the scheme for complex flood inundation conditions within a floodplain, the water quantity of vertical supply is treated as a source term $Q_{i}$. Substituting Equation (16) by Equations (27), (33), and (34), the numerical scheme at a time-step can be given as follows:

$$
\begin{aligned}
A_{i} \frac{H_{i, k+1}-H_{i, k}}{\Delta t} & =-\sum_{\beta=1}^{m} \frac{1}{4 A^{\beta}}\left[K_{i j}\left(H_{j}-H_{i}\right)\left(b_{i} b_{j}+c_{i} c_{j}\right)\right. \\
& \left.+K_{i m}\left(H_{m}-H_{i}\right)\left(b_{i} b_{m}+c_{i} c_{m}\right)\right]+Q_{i}
\end{aligned}
$$

Because geometries of the control volume in Equation (35) account for great proportions, they are included into variables like:

$$
\left\{\begin{array}{l}
Y_{i j}^{\beta}=-\frac{K_{i j}}{4 A^{\beta}}\left(b_{i} b_{j}+c_{i} c_{j}\right) \\
Y_{i m}^{\beta}=-\frac{K_{i m}}{4 A^{\beta}}\left(b_{i} b_{m}+c_{i} c_{m}\right)
\end{array}\right.
$$

Thus, Equation (35) can be simplified as:

$$
\begin{aligned}
& A_{i} \frac{H_{i, k+1}-H_{i, k}}{\Delta t} \\
& =\sum_{\beta=1}^{m}\left[Y_{i j}^{\beta}\left(H_{j}-H_{i}\right)+Y_{i m}^{\beta}\left(H_{m}-H_{i}\right)\right]+Q_{i}
\end{aligned}
$$

Therefore, the numerical scheme of the TFDM based on irregular triangular meshes at node $i$ has been obtained. Equation (37) can be solved either by either explicit or implicit methods. The implicit scheme can be obtained if water levels $H_{i}, H_{j}$ and $H_{m}$ on the right side of Equation (37) take values at the end of a time-step. The implicit scheme of the TFDM can be written as: 


$$
\begin{aligned}
& A_{i} \frac{H_{i, k+1}-H_{i, k}}{\Delta t} \\
& =\sum_{\beta=1}^{m}\left[Y_{i j}^{\beta}\left(H_{j, k+1}-H_{i, k+1}\right)\right. \\
& \left.\quad+Y_{i m}^{\beta}\left(H_{m, k+1}-H_{i, k+1}\right)\right]+Q_{i}
\end{aligned}
$$

Based on the characteristics of Equation (38), iterative techniques, for example, Successive Over-Relaxation (SOR) or Gauss-Seidal, are effective for obtaining solutions. In this paper, the Gauss-Seidal iterative method, given by Equation (39), is adopted as below:

$$
H_{i, k}^{(p+1)}=\frac{\sum_{\beta=1}^{m}\left[Y_{i j}^{\beta} H_{j, k}^{p(p+1)}+Y_{i m}^{\beta} H_{m, k}^{p(p+1)}\right]+\frac{A_{i} H_{i, k}}{\Delta t}+Q_{i}}{\sum_{\beta=1}^{m}\left[Y_{i j}^{\beta}+Y_{i m}^{\beta}\right]+\frac{A_{i}}{\Delta t}}
$$

where $p$ represents iterative times, $H_{i, k}^{(p+1)}$ will be the water level at the end of a time-step for node $i$, namely, $H_{i, k+1}$ when accuracy requirements of iterations are fulfilled. $H_{j, k}^{p(p+1)}$ and $H_{m, k}^{p(p+1)}$ mean that water levels at the iterative step $p+1$ will be used if they are known for vertices $j$ and $m$ of the triangle $\beta$. Otherwise, the values at the step $p$ could be substituted if convergence condition has not yet been satisfied.

\section{Case Application}

\subsection{Study Area}

A case study was conducted for the Fuefuki watershed with an area of $1,039.7 \mathrm{~km}^{2}$, which is bounded by Ara River and Fuefuki River with continuous levees. The study area and its elevation distribution are shown in Figure 5.

In this study, $\mathrm{x}$ is west-east direction while $\mathrm{y}$ represents south-north. The study area is divided into 891 grids on a $250 \mathrm{~m}$ DEM and has a total area of $58.31 \mathrm{~km}^{2}$. In order to make simulation results comparable with other methods, one mesh of the $250 \mathrm{~m}$ DEM was divided into two triangles by drawing a diagonal line. For the same study area, the number of triangular meshes obtained is 1781 with 963 nodes. The elevation distribution of the study region is shown in Figure 5. Based on maintenance conditions of the dikes along Fuefuki River, three locations (F110, F145 and $F 166)$ are assumed to be broken when suffering from serious flood events occurring in the basin. The general conditions at the assumed break sections are summarized in Table 1. In this paper, the inundation due to dike-break at $F 110$ is used as example to test the effectiveness of the proposed implicit TFDM numerical scheme.

\subsection{Treatment of waterfall or overflow}

Waterfall or overflow conditions may be present due to a

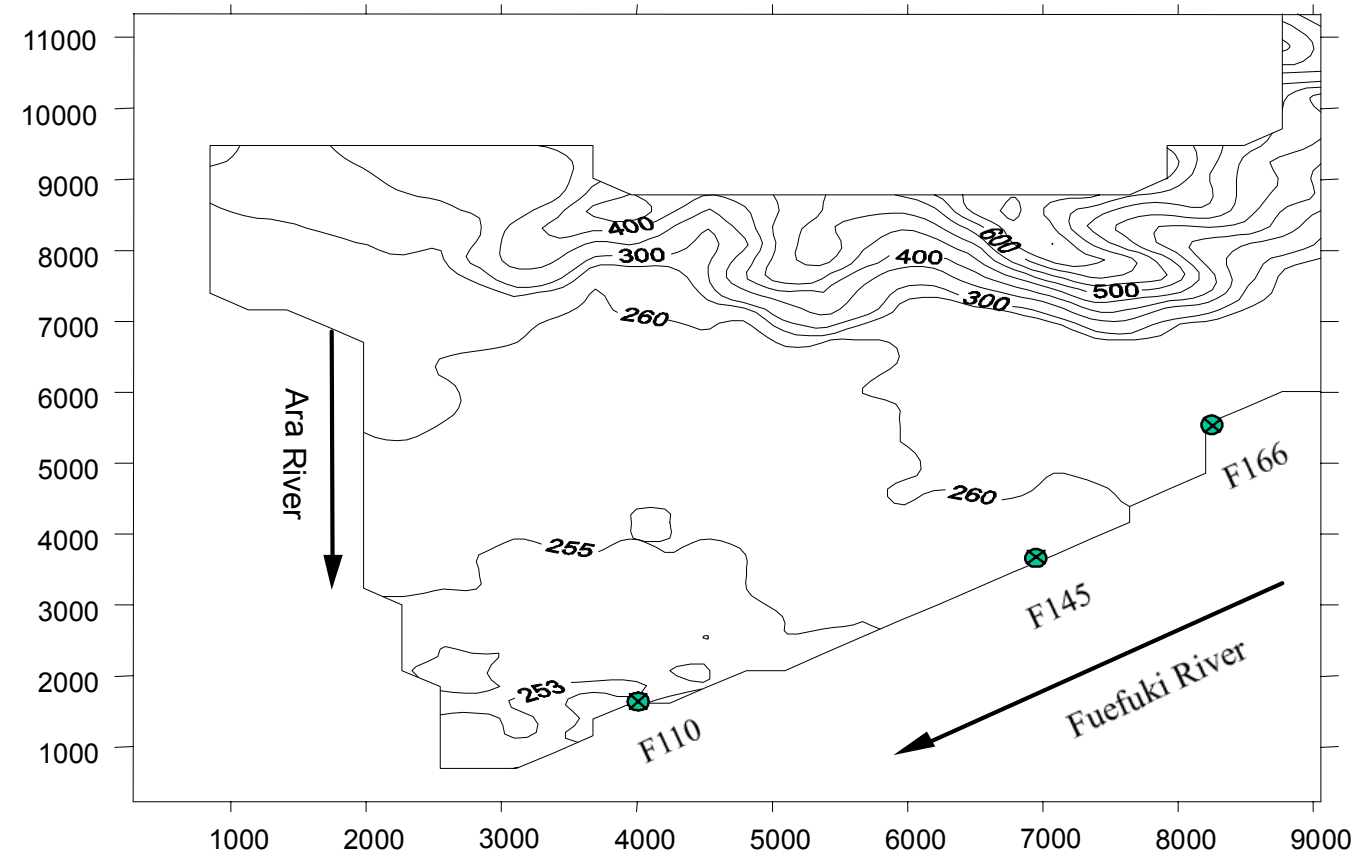

Figure 5. The Fuefuki Watershed and its elevation distribution. 
Table 1. Basic Conditions at Each Dike Breakage in the Study Area

\begin{tabular}{|c|c|c|c|c|c|c|c|c|c|c|c|}
\hline \multicolumn{4}{|c|}{ Locations of assumed dike-break } & \multicolumn{2}{|c|}{ Beginning of dike-break } & \multicolumn{6}{|c|}{ Flood probabilities (\%) and their peak discharges } \\
\hline No. & Sections & $\begin{array}{l}\text { Crest Elev. } \\
\text { (m) }\end{array}$ & $\begin{array}{l}\text { Ground } \\
\text { Elev. (m) }\end{array}$ & $\begin{array}{l}\text { Discharge } \\
\left(\mathrm{m}^{3} / \mathrm{s}\right)\end{array}$ & $\begin{array}{l}\text { Water } \\
\text { Level (m) }\end{array}$ & $\begin{array}{l}0.1 \% \\
6,700\end{array}$ & $\begin{array}{l}0.2 \% \\
6,000\end{array}$ & $\begin{array}{l}0.5 \% \\
5,200\end{array}$ & $\begin{array}{l}1.0 \% \\
3,600\end{array}$ & $\begin{array}{l}2.0 \% \\
3,100\end{array}$ & $\begin{array}{l}3.33 \% \\
2,500\end{array}$ \\
\hline 1 & F110 & 258.76 & 254.40 & 2,827 & 256.0 & $\bigcirc$ & $\bigcirc$ & $\bigcirc$ & O & O & $\times$ \\
\hline 2 & F145 & 263.26 & 259.60 & 2,881 & 261.4 & O & O & O & O & O & $\times$ \\
\hline 3 & F166 & 269.80 & 264.30 & 2,117 & 267.6 & 0 & $\bigcirc$ & 0 & 0 & 0 & $\bigcirc$ \\
\hline
\end{tabular}

Note: $\times=$ no dyke-break; $\bigcirc=$ dike-break

sudden change of ground elevation or, sometimes, of artificial structures in floodplains, as shown in Figure 6. During the simulation process, they have to be treated properly because of their discontinuity. In this study, the discontinuity is included in the calculation of the coefficient $K$. In Figure 3, for example, the flux can be estimated by a complete weir flow if there is a waterfall or overflow between node $i$ and $j$, as given below.

$q_{i, j}=\mu h_{1} \sqrt{2 g h_{1}} \quad$ if $h_{2} / h_{1} \leq 2 / 3$

or by a submerged weir flow

$q_{i, j}=\mu^{\prime} h_{2} \sqrt{2 g\left(h_{1}-h_{2}\right)} \quad$ if $h_{2} / h_{1}>2 / 3$

where $h_{l}$ and $h_{2}$ are the water depths in node $i$ and $j$, respectively. Please refer to Figure 6 for their definition. The weir flow coefficients $\mu$ and $\mu^{\prime}$ are treated as constant, and have a value of 0.35 and 0.91 , respectively.

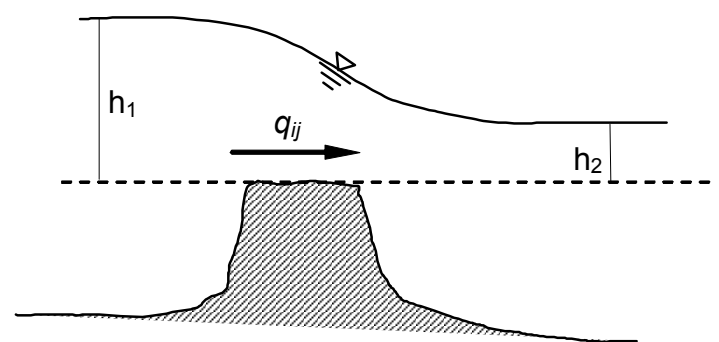

Figure 6. Waterfall or overflow situations.

The discharge flowing into the control volume at the node $i$ can be estimated by $q_{i j} \times L_{o j}$. Based Figure 3 and Equations (17), (18) and $\cot _{m}=20 j^{\prime} / i j$, the coefficient $K_{i, j}$ under this condition can be estimated by:

$K_{i j}=q_{i j} \cdot L_{i j} /\left(H_{j}-H_{i}\right)$ where $L_{i j}$ is the distance between the nodes $i$ and $j$. If this discontinuity occurs between the node $i$ and node $m, K_{i, m}$ can be obtained in the same way. Otherwise Equation (13) can be used to estimate $K_{i j}$, or $K_{i m}$.

\subsection{Boundary Condition}

In this study, the flood inundation due to dike break at the section $F 110$ was chosen to be the example for illustrating the effectiveness of the diffusive wave model and its implicit numerical scheme of the TFDM. It is supposed that a flood event with a probability of $1 \%$, generated on the basis of a historical flood occurring from August 14th to 16th of 1959, happened in the Fuefuki River basin and caused a dike break at the section F110. The hydrograph in the channel is shown in Figure 7(a), while Figure 7(b) is the inflow hydrograph from the break inlet. The flood inundation would begin when the water level in Fuefuki River reaches the H.W.L., which means that the discharge approaches $2,830 \mathrm{~m}^{3} / \mathrm{s}$ and the water level is $256.00 \mathrm{~m}$ at 7:45 a.m. as shown in Figure 7(a). The inflow hydrographs, as shown in Figure 7(b), are used as the input of boundary conditions.

\subsection{Initial condition}

For simplicity, the initial condition of simulation is assumed that the floodplain is dry before the dike-break at the section F110. Inundation water depths at all of the meshes of the study area are zero and the water surface elevations are equal to the ground surface elevations.

\subsection{Application Results}

The water level in the Fuefuki River channel reached $256.00 \mathrm{~m}$ and the discharge approached $2,830 \mathrm{~m}^{3} / \mathrm{s}$ at $7: 45$ a.m. on August 15, and the flood inundation lasted for 11 hours. The area change of inundation with time is plotted in Figure 8 and depicted by the line with rhombus dots. As indicated the figure, the variation of the inundated area appears not to be smooth. This is mainly because the elevation data of the $250 \mathrm{~m}$ DEM are in the form of integers. After the 11 hours of simulation, the extent of inundation or inundated areas in this flooding event finally stabilized at $12.21 \mathrm{~km}^{2}$, which accounts for $20.9 \%$ of the total area of study. The spatial distribution of inundated water depths is plotted in Figure 9(b). From the 


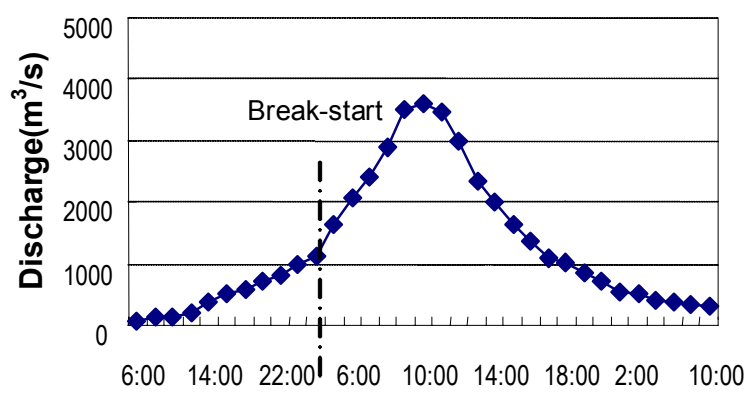

Aug. 14 Time(hour)

Aug. 16

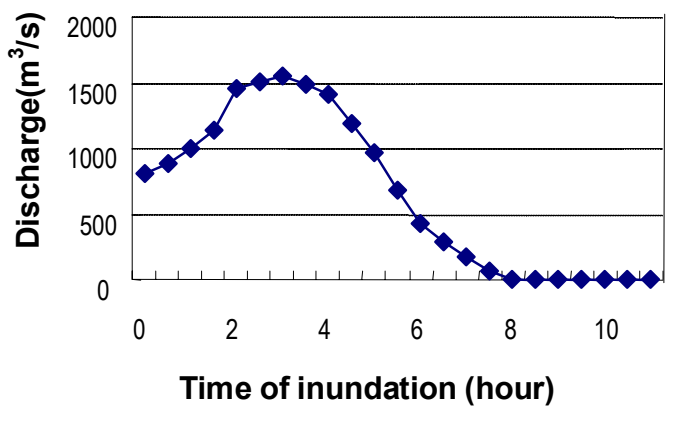

(b) The inflow hydrograph from the F110 break inlet

(a) The hydrographs for $1 \%$ probability

Figure 7. Flood hydrographs.

contour map of inundated water depths, as shown in Figure 9(b), majority of the inundation from the break inlet is located at the southwest lower region. In the small confluence region, where Ara River and Fuefuki River join together, the contour lines of water depths are densely distributed.

For the purpose of illustrating the variation of the inundation water depth, the representative location is selected at the lowest mesh point in the study region, which is one $250 \mathrm{~m}$ mesh north of the pump station with an elevation of $251.00 \mathrm{~m}$. The change of the water depth at this point is shown in Figure 10. The line with rectangular dots in the figure represents this variation. When the flood inundation ended at 18:45, the water depth reaches $5.02 \mathrm{~m}$.

\section{Comparisons and Discussions}

In order to testify the validity of the proposed $2 \mathrm{D}$ diffusive wave model for 2D inundation flows due to dike break, comparisons with other simulation methods have to be made because no observation is available for this kind of study. Available models for the comparison include 2D SLES (Iwasa et al., 1980), 2D RMA2, and FESWMS. The 2D RMA and FESWMS models are used to compute water surface elevations and flow velocities at nodes in a finite element mesh representing a body of water such as river, harbor, or estuary. The two models with the numerical simulation algorithm of FEM can analyze both steady and unsteady problems accurately. They can represent quite well arbitrary geometrical complexities of a study domain with different types of elements, consisting of quadrilateral, triangles and lines. However, the both models have disadvantages stemming from the discretization method of the FEM. The FEM conserves mass over an entire domain, but not within each element or at each node. The computational efforts needed by the FEM can be much greater than those for the FDM. In addition, spurious oscillations will be produced at flow discontinuities if special measures are not taken. Therefore, the simulation model of 2D SLES was adopted in this study for the purpose of comparison because it has been proved accurate and stable from practical applications in Japan. The algorithm of the 2D SLES has been widely used in flood inundation simulation in Japan. Owing to its high accuracy and stability, flood hazard maps and flood warning systems in most river basins in Japan have been established based on this scheme. The governing equations consist of the continuity equation (1) and the momentum equations (2) and (3) in $\mathrm{x}$ - and y- directions, respectively, as described in section 2 .

\subsection{Comparisons with the Simulated Results of the Com- plete 2D SVEs Model}

\subsubsection{Variations of Inundated Area}

Because the elevation of Ara River is higher than the ground elevation at its vicinity, and the Fuefuki River is confined by dikes within the confluence region, the drainage can only be carried out by a pump station as indicated in Figure 5. For simplicity, and the comparability of the proposed implicit TFDM with the 2D SLES, pumping and other drainage measures are temporarily not addressed in this paper because the primary objective of this paper is to verify the possibility of ignoring the inertia terms in the 2D SVEs, and the effectiveness of the proposed TFDM numerical scheme. Both the numerical methods of the TFDM and the 2D SLES use the same initial and boundary conditions as stated before. An identical time-step of iteration, which is 2 seconds, is used for the simulation. The total time of simulation is 11 hours for $1 \%$ flood event in the basin. The variations of inundated areas simulated by these two different methods are shown in Figure 8. Figure 9 shows the contour maps of inundated water depths at the end of the simulations.

Based on Figure 8, it is obvious that the change processes of inundated areas simulated by these two methods are almost the same, although the dynamic property mainly appears at the rising limb of the hydrograph. Since one mesh of the $250 \mathrm{~m} \mathrm{DEM}$ in this region has an area of $0.065 \mathrm{~km}^{2}$, the maximum discrepancy of simulated flooding area is $0.28 \mathrm{~km}^{2}$, and 


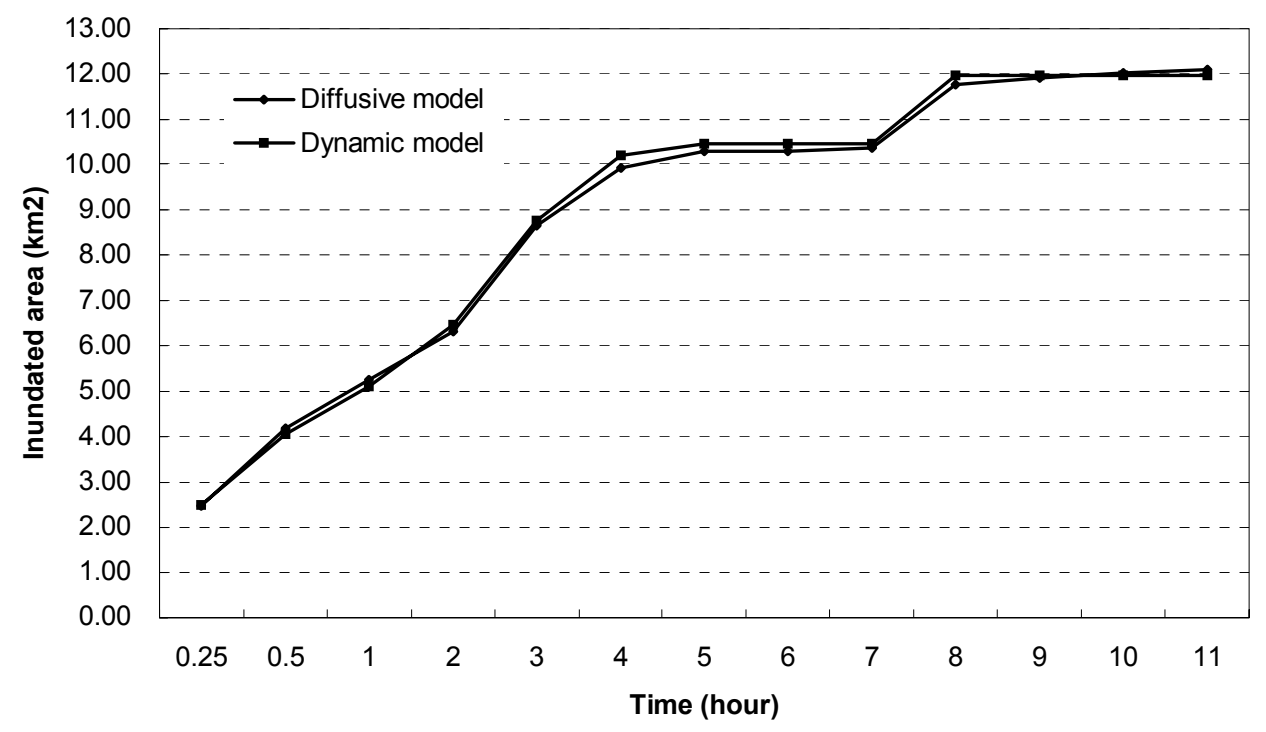

Figure 8. Variation of inundated areas.

covers approximately 4.5 meshes of the DEM. Only 3 percent of discrepancy has been found in the inundated areas. This indicates that the implicit TFDM numerical scheme of 2D diffusive wave model is reliable for estimation of inundation areas in floodplains.

\subsubsection{Inundated Water Depth Variation}

The effectiveness of the implicit TFDM cannot be tested sufficiently for inundation simulation only by analyzing inundated area variation. Inundated water depth and its spatial distribution are also important indexes for damage assessment. It is impossible to give inundated water depths of all submerged meshes. However, the efficiency can be judged based on the spatial distribution of inundated water depths as shown by the contour maps in Figure 9. In order to demonstrate the effectiveness of the proposed method from this perspective, the representative location is selected at the same lowest mesh, which is one $250 \mathrm{~m}$ mesh north of the pump station, where the elevation is $251.00 \mathrm{~m}$. The changes of inundated water depth with the time change are displayed in Figure 10. As indicated in this figure, the simulated result by the implicit TFDM is approximately equal to that of the complete 2D SVE with 2D SLES numerical scheme. The discrepancy in inundated water depths appears mostly before the peak time of inflow hydrograph from the broken inlet. The maximum discrepancy is $0.32 \mathrm{~m}$, which accounts for $6.4 \%$ of the maximum water depth of $5.02 \mathrm{~m}$ in the simulation. The averaged discrepancy is -0.02 $\mathrm{m}$, which is not significant. Based on the water depth variation as shown in Figure 10, the spatial distributions of water depths, and the contour map as shown in Figure 9, the result shows that the proposed method is also reliable in the estimation of inundated water depth.

\subsection{Discussions}

\subsubsection{Difficulties in the Verification of Inundation Models}

Because flood inundation due to dike-break is a flooding event occurring at an extreme condition of emergency, there are no observed data available. Among the traditional approaches including field measurements, laboratory experiments, and depth-averaged computer models, only the application of computer models is the feasible for this study. However, the efficiency of the numerical models can hardly be verified based on conventional methods of comparing simulations with observations. Comparisons among different models should be a dependable tactic for the purpose of testifying the effectiveness of the proposed models.

Hromadka and Yen (1986) used diffusion hydrodynamic model to deal with dam-break and other problems. Based on their study, the diffusive wave model can accurately approximate complete 1D SVEs for one-dimensional dam-break problem. The difference in predicted water depths are within $3 \%$ when compared with the U.S.G.S fully dynamic K-634 dam-break model. But for the 2D diffusive wave model, Xanthopoulos and Koutitas (1976) conceptually verified it by considering evolution of inundated area, which propagates radially from dam-break site. Hromadka and Yen (1986) concluded that the 2D DHM might be justifiable based on small differences in predicted flooding depths of 1D dam-break simulation. 


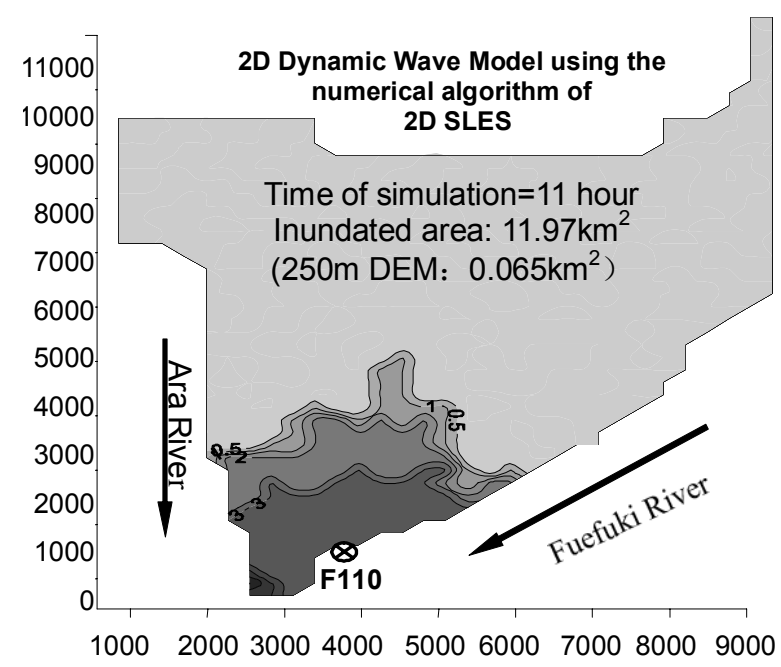

(a) Spatial distribution of inundated water depths simulated by the complete 2D SVEs with the numerical algorithm of the 2D SLES

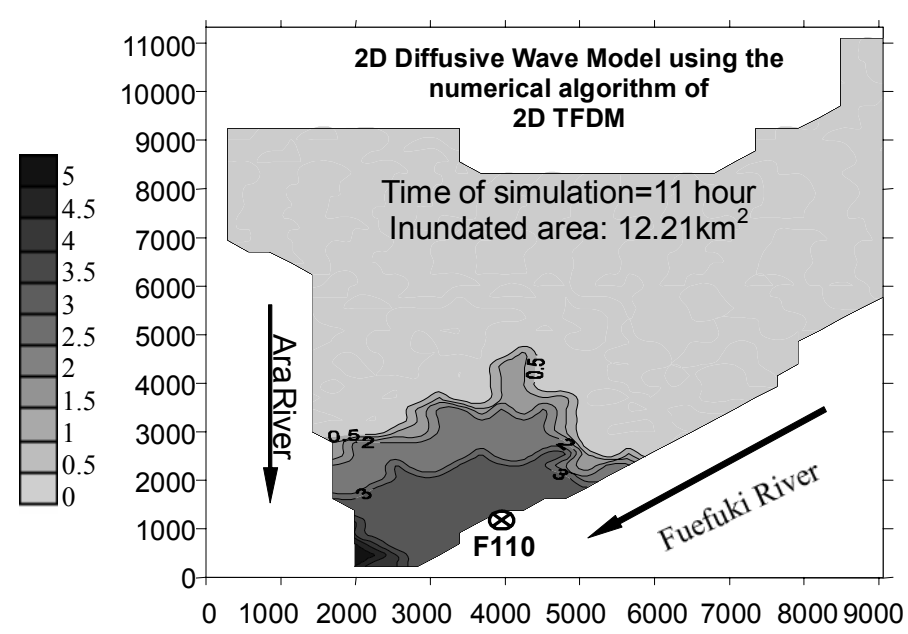

(b) Spatial distribution of inundated water depths simulated by the $2 \mathrm{D}$ diffusive wave model with the numerical algorithm of the 2D TFDM

Figure 9. Inundated water depths simulated by two different models.

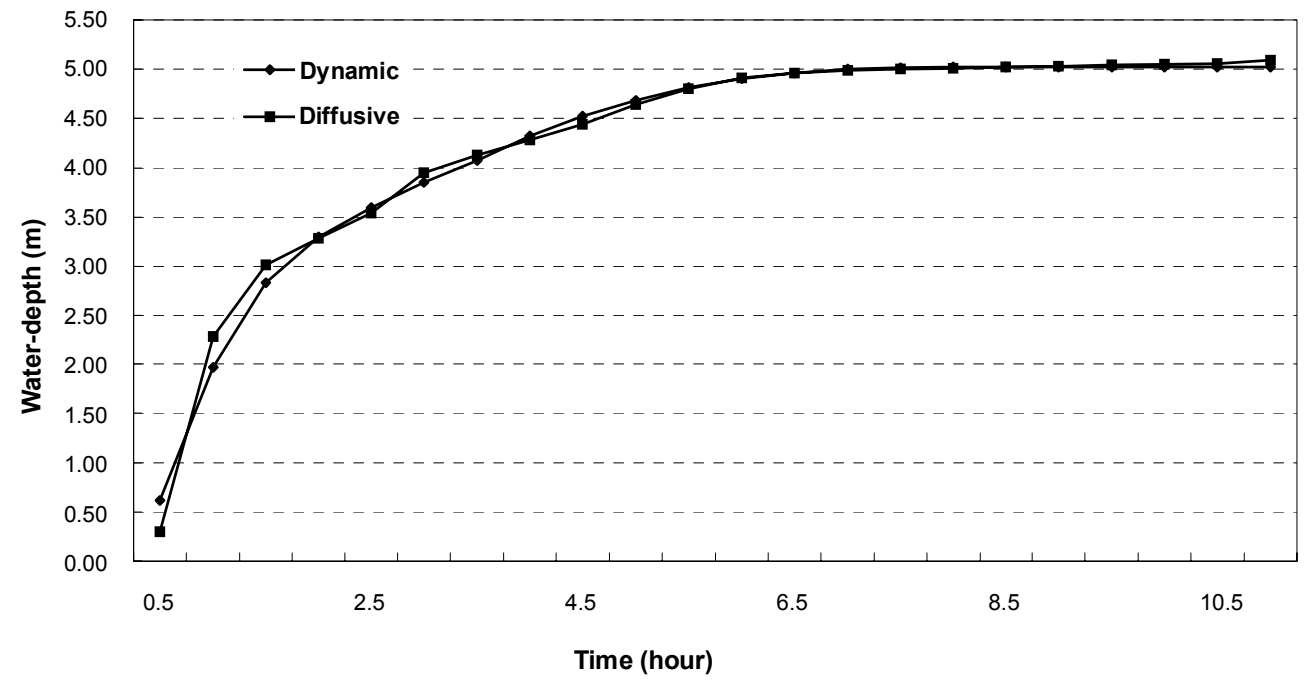

Figure 10. Inundated water depth variations at the lowest grid point. 


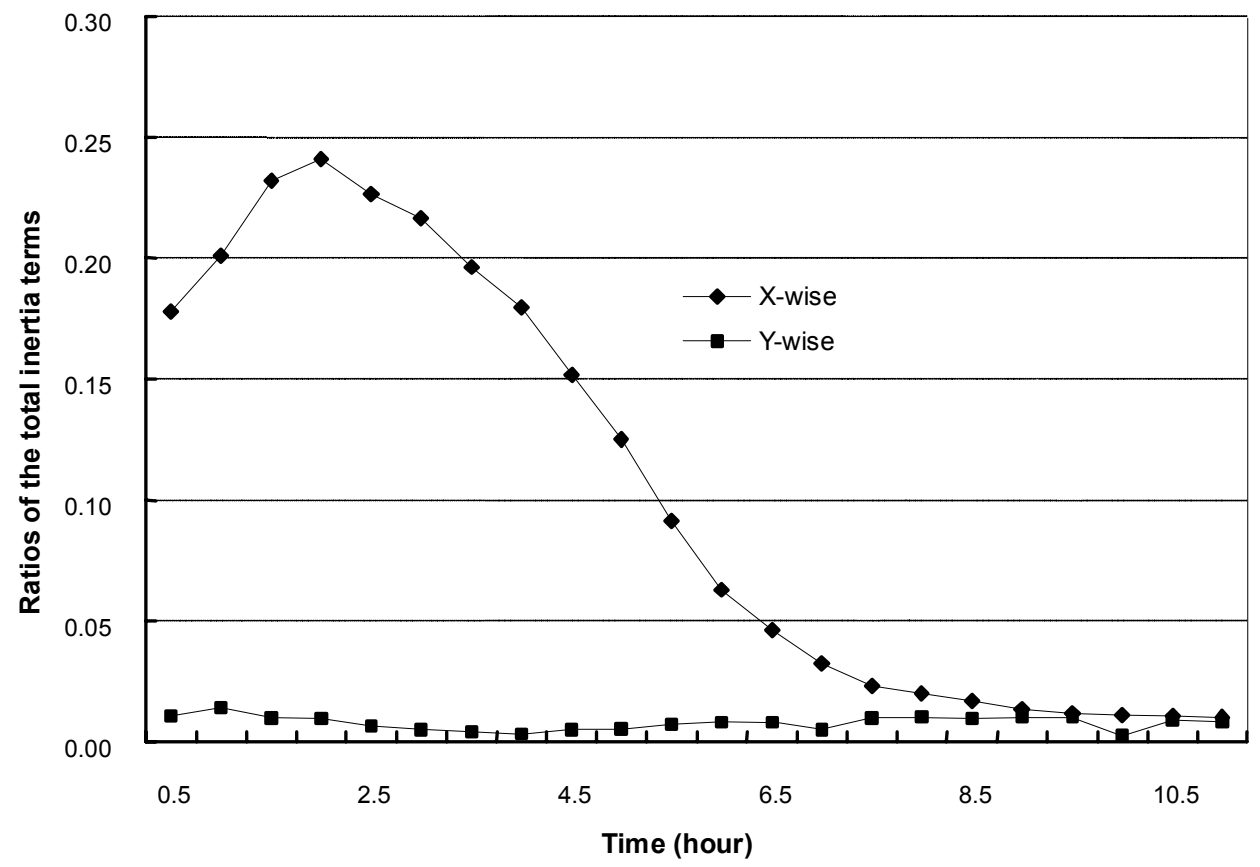

Figure 11. Ratio variations of the total inertia terms in the momentum equations.

De Roo et al. (2000) used an explicit finite difference scheme based on 2D Kinematic wave model to simulate flood inundations occurred in January 1995 on the floodplain of the Rive Meuse between the gauging stations at Borgharen in the Netherlands and Maaseik in Belgium. The air photo and Synthetic Aperture Rada (SAR) data are available for the flood inundation in 1995. Compared with these observed photo data, their results of simulation shown an $85.5 \%$ agreement.

As noted above, the proposed 2D diffusive wave model discretized by the implicit TFDM scheme in this study can approximate the complete 2D SVEs with reliable accuracy, which demonstrates that the discrepancies of extents, and water depths simulated by these two different models are 3\% and $6.4 \%$, respectively. 2D diffusive wave model can upgrade the accuracy of simulation by approximately $10 \%$ from the 2D kinematic wave model, used by De Roo et al. (2000).

\subsubsection{Ratios of Three Inertia Terms in the Whole Momentum} Equations

The simulated results have illustrated the effectiveness of the proposed 2D diffusive wave model and its numerical scheme for the inundation simulation. How much the quantity of the three inertia terms on the left-hand sides of the equations (2) and (3) accounts for in the total five terms of momentum equations are not yet clear. Few papers have ever investigated into this subject. For this purpose, we tried in this study to ascertain it by assuming that the results obtained by the 2D SLES scheme for 2D SVEs are correct. Because inundation flow direction from the break-inlet, either in $\mathrm{x}$ - or $\mathrm{y}$-directions, must be assigned in advance for this numerical scheme, minus $\mathrm{x}$-direction is determined as the needed direction based on the direction of water flow in the Fuefuki River channel. Therefore, the inertia terms in $\mathrm{x}$ - and $\mathrm{y}$-directions can be computed separately. The statistical ratios of the three terms in the total five terms of the momentum equations can be obtained in the simulation processes. The ratio changes of the three inertia terms with the time change are depicted in Figure 11.

As shown in Figure 11, the ratios of the three inertia terms in $\mathrm{x}$-direction, which is the inflow direction of inundation flow from the $F 110$ break inlet, are relatively large and mainly exist before and around the flood peak of the inflow. For example, more than $15 \%$ of inertia ratios appear before 4.5 hours of inundation, during which the discharges of incoming flood flow are over $1,000 \mathrm{~m}^{3} / \mathrm{s}$. The maximum ratio $24.08 \%$ appeared at 2 hours of inundation, where change of discharge is substantial as shown in Figure 7(b). This situation is analogous to 1D dam-break problems, in which Hromadka and Yen (1986) concluded that $1 \mathrm{D}$ diffusive wave model could approximate the 1D SVE with $97 \%$ accuracy in the predication of water depths. However, in the other direction, the y-direction, it is generally smaller than $1.0 \%$ and completely negligible. The maximum inertia ratio is only $1.5 \%$. Their statistical values in 
both directions are summarized in Table 2. The averaged magnitudes of these ratios in $\mathrm{x}$ - and $\mathrm{y}$-directions are $12.5 \%$ and $0.85 \%$, respectively. This indicates that the inertia terms in the 2D SVEs are not significant and can be neglected.

Table 2. Ratios of Inertia Terms in the Momentum Equations

\begin{tabular}{lllllll}
\hline \multirow{2}{*}{$\begin{array}{l}\text { Dike-break } \\
\text { direction }\end{array}$} & \multicolumn{3}{c}{ Ratios in X-direction } & \multicolumn{3}{c}{ Ratios in Y-direction } \\
& Max. & Min. & Ave. & Max. & Min. & Ave. \\
\hline $\mathrm{X}$ & 0.2408 & 0.0106 & 0.1247 & 0.0147 & 0.0028 & 0.0085 \\
\hline
\end{tabular}

\section{Concluding Remarks}

In this study, a diffusive wave model was proposed for flood inundation simulation due to dike-break. Considering disadvantages of the uniform rectangular grid system used in the finite difference schemes, a TFDM numerical algorithm based on irregular triangle meshes is developed in this study, integrating complex land uses, topography, and irregular geometries of inner and outer boundary conditions into the simulation of flood inundation. A case study was carried out in the Fuefuki River basin in central Japan. In order to testify the effectiveness of the proposed diffusive model and its numerical scheme, the complete 2D SVEs with an accurate numerical algorithm of 2D SLES developed by IWSA (1980), was used for comparison in the case study. Results indicate that the discrepancies of simulated inundation areas and water depths between the two methods are $3.0 \%$ and $6.4 \%$, respectively. In addition, an analysis on the inertia terms in the momentum equations was also performed in this study. In x-direction of the assumed dike-break direction, the inertia terms account for an average of $12.5 \%$ of the total five terms of the momentum equations, while in the y-direction, the inertia terms are insignificant with only $0.85 \%$. Although the inertia terms account for relatively bigger proportions in the whole momentum equations, their effects finally contribute to spatial distribution of inundated water depths and extents of inundation. The actual effects should be evaluated based on simulation results of inundated areas and distribution of water depths. Furthermore, the 2D diffusive wave model can improve the accuracy of $2 \mathrm{D}$ kinematic wave model by approximately $10 \%$, based on the result of De Roo et al. (2000).

The implicit TFDM numerical scheme of the 2D diffusive wave model is a simple and relatively accurate scheme with its unique features. Irregular triangle meshes allow geometrical influences of boundaries and artificial structures within a floodplain to be integrated into simula- tion process. The implicit numerical scheme permits time-step to be reasonably enlarged without experiencing the puzzling situation of instability if there is a need for longer time simulation. As well, this model solves the integral form of the governing 2D diffusive wave equation in each computational cell. Thus, mass and momentum can be conserved much well than in those models based on the FEM. It is suggested that the diffusive wave model and its implicit TFDM numerical scheme developed in this study are an effective and reliable approach to assessing flood inundation due to dike break.

\section{References}

Aderson, W.K. and Bonhaus, D.L. (1994). An implicit upwind algorithm for computing turbulent flows on unstructured grids. Comput. Fluids, 23(1), 1-21.

Aizinger, V. and Dawson, C. (2002). A discontinuous galerkin method for two-dimensional flow and transport in shallow water Adv. Water Resour., 25, 67-84.

Akan, A.O. and Yen, B.C. (1981). Diffusion-wave routing in channel networks. J. Hydraul. Div., 107(HY6), 719-732.

De Roo, A.P.J., Van Der Knijff, J., Schmuck, G. and Bates, P. (2000). A simple floodplain inundation model to assist in floodplain manegement, in U. Maione, B. Majone-Lehto and R. Monti (Eds.), New Trends in Water and Environmental Engineering for Safety and Life, Balkema, Rotterdam, ISBN 9058091384, pp. $1-10$.

Fennema, R.J., Neidrauer, C.J., Johnson, R.A., MacVicar, T.K. and Perkins, W.A. (1994). A computer model to simulate natureal everglades hydrology, in S.M. Davis and J.C. Ogden (Eds.), Everglades, the Ecosystem and its Restoration. St. Lucie Press, Florida, USA, pp. 249-289.

Holmes, D.G. and Connell, S.D. (1989). Solution of the 2D navier-stokes equations on unstructured adaptive grids. $A I A A P a-$ per 89-1932-CP, 25-39.

Hromadka II, T.V. and Lai, C. (1985). Solving the two-dimensional diffusion flow model, in Proc. of the Specialty Conference, Sponsored by the Hydraulic Division of ASCE, Lake Buena Vista, Florida, USA, pp. 555-561.

Hromadka II, T.V., Walker, T.R., Yen, C.C. and Devries, J.J. (1989). Application of the U.S.G.S. diffusive wave model for urban floodplain analysis. Water Resour. Bull., 25(5), 1063-1071.

Hromadka II, T.V. and Durbin T.J. (1986). Two-dimensional dam-break flood-flow analysis for orange county reservoir. $\mathrm{Wa}$ ter Resour. Bull., 22(2), 257-265.

Hromadka II, T.V. and Yen, C.C. (1986). A diffusion hydrodynamic model (DHM). Adv. Water Resour., 9(3), 118-170.

Iwasa, Y., Inoue, K. and Mizutori, M. (1980). Hydraulic analysis of overland flood flows by means of numerical method. Annuals of Disaster Prevention Research Institute, 23(B-2) 305-317. (in Japanese).

Lal A.M.W. (1998). Performance comparison of overland flow algorithms. J. Hydraul. Eng., 124(4), 342-349.

Lal A.M.W. (2000). Numerical errors in groundwater and overland flow models. Water Resour. Res., 36(5), 1237-1247.

Ponce, V. M., Li, R.M. and Sinons, D.B. (1978). Applicability of kinematic and diffusion waves. J. Hydraul. Div., 104(3), 353-360.

Suetsugi, T. and Kuriki, M. (1998). Research on application for flood disaster prevention and simulation of flooding flow by means of new flood simulation model. J. Hydraul. Coast. Environ. Eng., Japan, 593/II-43, 41-50. (in Japanese).

Xanthopoulos, T. and Koutitas, C. (1976). Numerical simulation of a two-dimensional flood wave propagation due to dam-failure. $J$. Hydraul. Res., 14(4), 321-331. 